\section{OPEN $\partial$ ACCESS}

\title{
Crystal structure of 3-(2,5-dimethoxy- phenyl)propionic acid
}

\author{
Bernhard Bugenhagen, ${ }^{a}$ Yosef Al Jasem, ${ }^{\mathrm{b}}$ Mariam AlAzani ${ }^{\mathrm{c}}$ \\ and Thies Thiemann ${ }^{\mathrm{C} *}$
}

anstitute of Inorganic Chemistry, University of Hamburg, Hamburg, Germany,

${ }^{\mathbf{b}}$ Department of Chemical Engineering, and ${ }^{\mathbf{c}}$ Department of Chemistry, United Arab Emirates University, AL Ain, Abu Dhabi, United Arab Emirates. *Correspondence

e-mail: thies@uaeu.ac.ae

Received 12 April 2015; accepted 18 April 2015

Edited by P. McArdle, National University of Ireland, Ireland

In the crystal of the title compound, $\mathrm{C}_{11} \mathrm{H}_{14} \mathrm{O}_{4}$, the aromatic ring is almost coplanar with the 2-position methoxy group with which it subtends a dihedral of $0.54(2)^{\circ}$, while the 5-position methoxy group makes a corresponding dihedral angle of just $5.30(2)^{\circ}$. The angle between the mean planes of the aromatic ring and the propionic acid group is $78.56(2)^{\circ}$. The fully extended propionic side chain is in a trans configuration with a $\mathrm{C}-\mathrm{C}-\mathrm{C}-\mathrm{C}$ torsion angle of $-172.25(7)^{\circ}$. In the crystal, hydrogen bonding is limited to dimer formation via $R_{2}{ }^{2}(8)$ rings. The hydrogen-bonded dimers are stacked along the $b$ axis. The average planes of the two benzene rings in a dimer are parallel to each other, but at an offset of 4.31 (2) $\AA$. Within neighbouring dimers along the [101] direction, the average molecular benzene planes are almost perpendicular to each other, with a dihedral angle of $85.33(2)^{\circ}$.

Keywords: crystal structure; 3-(2,5-dimethoxyphenyl)propionic acid; O$\mathrm{H}$... O hydrogen bonding.

CCDC reference: 1060285

\section{Related literature}

For another preparation method of the title compound, see: Anliker et al. (1957). For crystal structures of phenylpropionic acids, see: Das et al. (2012). For the application of the title compound as a starting material for 19-norsteroidal derivatives, see: Anliker et al. (1957); and as a starting material for amidoethylquinones, see: Bremer et al. (2014).

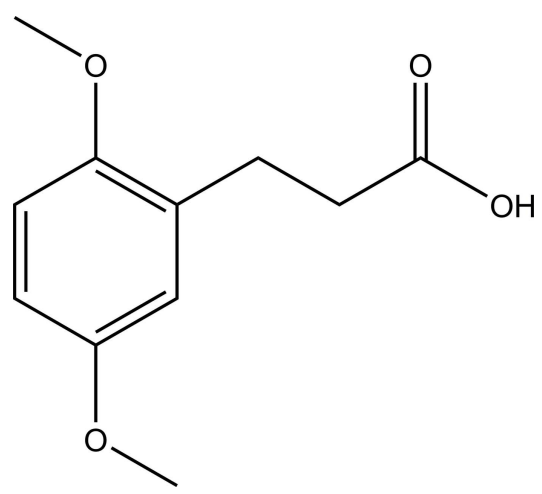

2. Experimental

2.1. Crystal data

$\mathrm{C}_{11} \mathrm{H}_{14} \mathrm{O}_{4}$

$M_{r}=210.22$

Monoclinic, $C 2 / c$

$a=24.3212(10) \AA$

$b=4.6512(2) \AA$

$c=19.7411(8) \AA$

$\beta=109.1782(6)^{\circ}$

$$
\begin{aligned}
& V=2109.23(15) \AA^{3} \\
& Z=8 \\
& \text { Mo } K \alpha \text { radiation } \\
& \mu=0.10 \mathrm{~mm}^{-1} \\
& T=100 \mathrm{~K} \\
& 0.3 \times 0.1 \times 0.02 \mathrm{~mm}
\end{aligned}
$$

\subsection{Data collection}

Bruker APEXII CCD diffractometer

Absorption correction: multi-scan (SADABS; Bruker, 2013)

$T_{\min }=0.604, T_{\max }=0.746$

\subsection{Refinement}

$R\left[F^{2}>2 \sigma\left(F^{2}\right)\right]=0.036$

$w R\left(F^{2}\right)=0.107$

$S=1.05$

3224 reflections

142 parameters

20284 measured reflections 3224 independent reflections 2927 reflections with $I>2 \sigma(I)$ $R_{\text {int }}=0.028$

Table 1

Hydrogen-bond geometry $\left(\AA{ }^{\circ}\right)$.

\begin{tabular}{lcclc}
\hline$D-\mathrm{H} \cdots A$ & $D-\mathrm{H}$ & $\mathrm{H} \cdots A$ & $D \cdots A$ & $D-\mathrm{H} \cdots A$ \\
\hline $\mathrm{O}^{2}-\mathrm{H} 4 \cdots \mathrm{O}^{\mathrm{i}}$ & $0.92(2)$ & $1.75(2)$ & $2.6624(11)$ & $172.1(18)$ \\
\hline Symmetry code: (i) & $-x+1,-y+2,-z+1$.
\end{tabular}

Data collection: APEX2 (Bruker, 2013); cell refinement: SAINT (Bruker, 2013); data reduction: $S A I N T$; $\operatorname{program}(\mathrm{s})$ used to solve structure: SIR2004 (Burla et al., 2007); program(s) used to refine structure: SHELXL2013 (Sheldrick, 2015); molecular graphics: PLATON (Spek, 2009) and Mercury (Macrae et al., 2008); software used to prepare material for publication: OLEX2 (Dolomanov et al., 2009).

\section{Acknowledgements}

MA thanks the UAEU for a PhD scholarship. 


\section{data reports}

Supporting information for this paper is available from the $\mathrm{IUCr}$ electronic archives (Reference: QM2110).

\section{References}

Anliker, R., Lindsey, A. S., Nettleton, D. E. \& Turner, R. B. Jr (1957). J. Am. Chem. Soc. 79, 220-226.

Bremer, P. T., Hixon, M. S. \& Janda, K. D. (2014). Bioorg. Med. Chem. 22 , 3971-3981.

Bruker (2013). APEX2, SAINT and SADABS. Bruker AXS Inc., Madison, Wisconsin, USA.
Burla, M. C., Caliandro, R., Camalli, M., Carrozzini, B., Cascarano, G. L., De Caro, L., Giacovazzo, C., Polidori, G., Siliqi, D. \& Spagna, R. (2007). J. Appl. Cryst. 40, 609-613.

Das, U., Chattopadhyay, B., Mukherjee, M. \& Mukherjee, A. K. (2012). Cryst. Growth Des. 12, 466-474.

Dolomanov, O. V., Bourhis, L. J., Gildea, R. J., Howard, J. A. K. \& Puschmann, H. (2009). J. Appl. Cryst. 42, 339-341.

Macrae, C. F., Bruno, I. J., Chisholm, J. A., Edgington, P. R., McCabe, P., Pidcock, E., Rodriguez-Monge, L., Taylor, R., van de Streek, J. \& Wood, P. A. (2008). J. Appl. Cryst. 41, 466-470.

Sheldrick, G. M. (2015). Acta Cryst. C71, 3-8.

Spek, A. L. (2009). Acta Cryst. D65, 148-155. 


\title{
supporting information
}

Acta Cryst. (2015). E71, o337-o338 [https://doi.org/10.1107/S2056989015007641]

\section{Crystal structure of 3-(2,5-dimethoxyphenyl) propionic acid}

\author{
Bernhard Bugenhagen, Yosef Al Jasem, Mariam AlAzani and Thies Thiemann
}

\section{S1. Structural commentary}

The molecule of the title compound exhibits one conformation (Figure 1), unlike other analogous compounds that exhibit two conformations (e.g. 3-phenylpropionic acid, 3-(3-methylphenyl)propionic acid and 3-(3-methoxyphenyl)propionic acid) (Das et al., 2012). The aromatic ring of the title compound is almost coplanar with the $\mathrm{C} 10$ methoxyl with which it has a dihedral of less than 0.54 (2) ${ }^{\circ}$ while the $\mathrm{C} 11$ methoxyl has a corresponding dihedral of just $5.30(2)^{\circ}$. The angle between the mean planes of the aromatic ring and the propionic acid group $(\mathrm{C} 7, \mathrm{C} 8, \mathrm{C} 9, \mathrm{O} 3$ and $\mathrm{O} 4)$ is $78.56(2)^{\circ}$. The fully extended propionic side chain is in a trans configuration with $(\mathrm{C} 6-\mathrm{C} 7-\mathrm{C} 8-\mathrm{C} 9)$ torsion angle of $-172.25(2)^{\circ}$. The $\mathrm{O} 4-\mathrm{H} 4 \cdots \mathrm{O} 3$ hydrogen bonding (Table 1 ) of the $\mathrm{COOH}$ functional groups leads to dimer formation via $R^{2}{ }_{2}(8)$ rings. The hydrogen bonded dimers are stacked along the $b$ axis. The average planes of the two benzene rings in a dimer are parallel to each other, but at an offset of 4.31 (2) $\AA$. Within neighboring dimers along [101] direction, the average molecular benzene planes are almost perpendicular to each other, with an angle of $85.33(2)^{\circ}$. No other appreciable close contacts were noticed except a very weak $\mathrm{C} 3-\mathrm{H} 3 \cdots \pi$ interaction between adjacent dimers along [101], with a bond length of 3.20 (2) A.

\section{S2. Synthesis and crystallization}

3-(2,5-Dimethoxyphenyl)propionic acid. - Ethyl 3-(2,5-dimethoxyphenyl)propionate (3.2 g, $13.4 \mathrm{mmol})$ in a mixture of aq. $\mathrm{NaOH}(10 \mathrm{w} \%, 30 \mathrm{~mL})$ and methanol $(8 \mathrm{~mL})$ was heated at reflux for $12 \mathrm{~h}$. Then, half. conc. aq. $\mathrm{HCl}$ is added to the cooled solution. Thereafter, the mixture is extracted with chloroform $(3 \mathrm{X} 15 \mathrm{~mL})$. The organic phase is dried over anhydrous $\mathrm{MgSO}_{4}$ and concentrated in vacuo. The residue is filtered over a small column of silica gel (diethylether$\left.\mathrm{CHCl}_{3}, 1: 1, \mathrm{v} / \mathrm{v}\right)$ to give the title compound ( $2.56 \mathrm{~g}, 89 \%$ ) as colorless needles, mp. 339 - $340 \mathrm{~K}$ [Lit. mp. 339-340 K (Anliker et al., 1957)]; $v_{\max }\left(\mathrm{KBr} / \mathrm{cm}^{-1}\right) 3500-2050$ (bs, OH), 2955, 2835, 1699, 1504, 1449, 1430, 1307, 1281, 1182, $1127,927,916,865,795,717,499 ; \delta_{\mathrm{H}}\left(400 \mathrm{MHz}, \mathrm{CDCl}_{3}\right) 2.65\left(2 \mathrm{H}, \mathrm{t},{ }^{3} J=7.6 \mathrm{~Hz}\right), 2.91\left(2 \mathrm{H}, \mathrm{t},{ }^{3} J=7.6 \mathrm{~Hz}\right), 6.71(1 \mathrm{H}$, $\left.\mathrm{dd},{ }^{3} J=8.4 \mathrm{~Hz},{ }^{4} J=3.2 \mathrm{~Hz}\right), 6.75\left(1 \mathrm{H}, \mathrm{d},{ }^{4} J=3.2 \mathrm{~Hz}\right), 6.76\left(1 \mathrm{H}, \mathrm{d},{ }^{3} J=8.4 \mathrm{~Hz}\right), \delta_{\mathrm{C}}\left(67.8 \mathrm{MHz}, \mathrm{CDCl}_{3}\right) 26.0\left(\mathrm{CH}_{2}\right), 33.9$ $\left(\mathrm{CH}_{2}\right), 55.6\left(\mathrm{OCH}_{3}\right), 55.7\left(\mathrm{OCH}_{3}\right), 111.0(\mathrm{CH}), 111.6(\mathrm{CH}), 116.3(\mathrm{CH}), 129.6(\mathrm{CH}), 151.7\left(\mathrm{C}_{\text {quat }}\right), 153.3\left(\mathrm{C}_{\text {quat }}\right), 179.7$ $\left(\mathrm{C}_{\text {quat }}, \mathrm{CO}\right)$.

\section{S3. Refinement}

All hydrogen atoms were placed in calculated positions with $\mathrm{C}-\mathrm{H}$ distances of $0.95-0.99 \AA$ and refined as riding with $U_{\text {iso }}(\mathrm{H})=\mathrm{x} U_{\text {eq }}(\mathrm{C})$, where $\mathrm{x}=1.5$ for methyl and $\mathrm{x}=1.2$ for all other $\mathrm{H}$-atoms. 


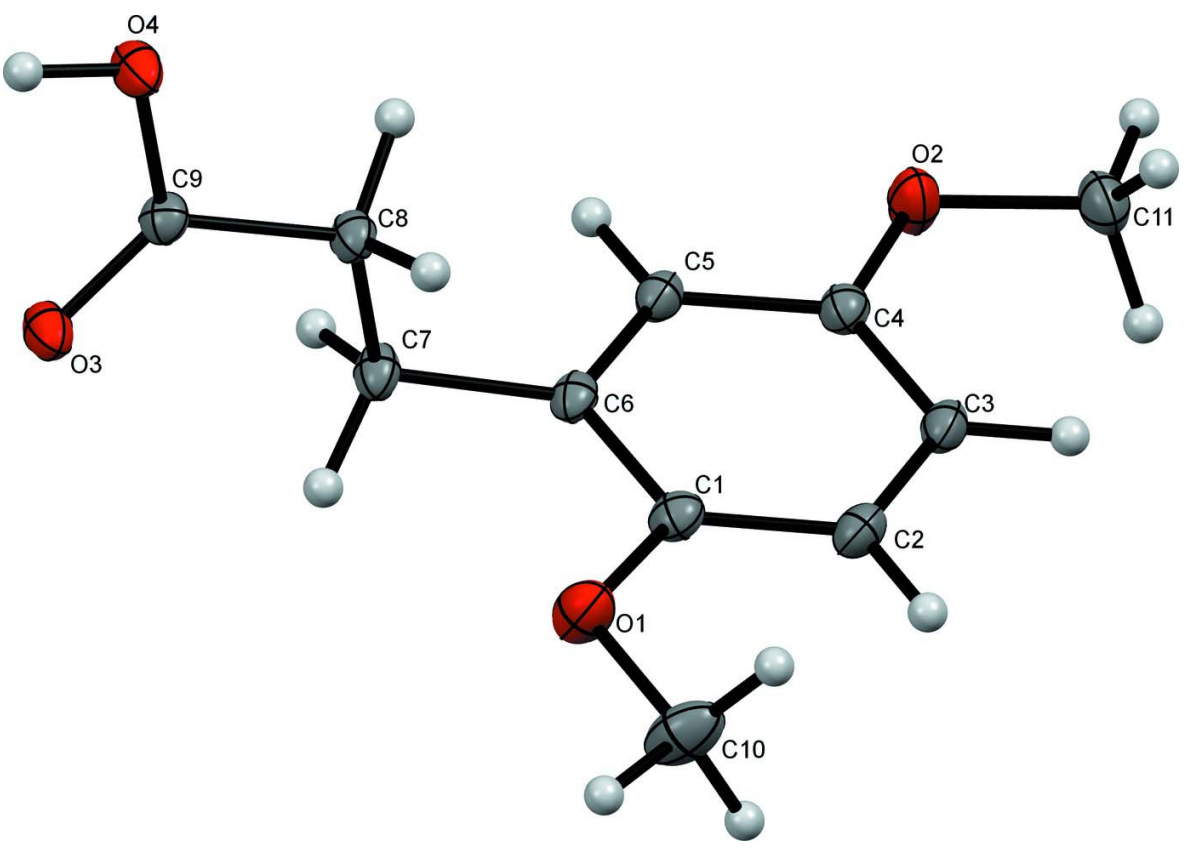

Figure 1

A view of title compound molecule with the atom-numbering scheme. Displacement ellipsoids are shown at the $50 \%$ probability level.

\section{3-(2,5-Dimethoxyphenyl)propionic acid}

Crystal data

$\mathrm{C}_{11} \mathrm{H}_{14} \mathrm{O}_{4}$

$M_{r}=210.22$

Monoclinic, $C 2 / c$

$a=24.3212(10) \AA$

$b=4.6512(2) \AA$

$c=19.7411(8) \AA$

$\beta=109.1782(6)^{\circ}$

$V=2109.23(15) \AA^{3}$

$Z=8$

$F(000)=896$

Data collection

Bruker APEXII CCD

diffractometer

$\varphi$ and $\omega$ scans

Absorption correction: multi-scan

(SADABS; Bruker, 2013)

$T_{\min }=0.604, T_{\max }=0.746$

20284 measured reflections

Refinement

Refinement on $F^{2}$

Least-squares matrix: full

$R\left[F^{2}>2 \sigma\left(F^{2}\right)\right]=0.036$

$w R\left(F^{2}\right)=0.107$

$S=1.05$
$D_{\mathrm{x}}=1.324 \mathrm{Mg} \mathrm{m}^{-3}$

Melting point $=339-340 \mathrm{~K}$

Mo $K \alpha$ radiation, $\lambda=0.71073 \AA$

Cell parameters from 9914 reflections

$\theta=2.3-31.2^{\circ}$

$\mu=0.10 \mathrm{~mm}^{-1}$

$T=100 \mathrm{~K}$

Bar, clear light colourless

$0.3 \times 0.1 \times 0.02 \mathrm{~mm}$

3224 independent reflections

2927 reflections with $I>2 \sigma(I)$

$R_{\text {int }}=0.028$

$\theta_{\text {max }}=31.3^{\circ}, \theta_{\min }=1.8^{\circ}$

$h=-35 \rightarrow 34$

$k=-6 \rightarrow 6$

$l=-27 \rightarrow 28$

3224 reflections

142 parameters

0 restraints

Primary atom site location: structure-invariant direct methods 
Hydrogen site location: mixed

$\mathrm{H}$ atoms treated by a mixture of independent and constrained refinement

$$
\begin{aligned}
& w=1 /\left[\sigma^{2}\left(F_{\mathrm{o}}{ }^{2}\right)+(0.0556 P)^{2}+1.4383 P\right] \\
& \text { where } P=\left(F_{\mathrm{o}}^{2}+2 F_{\mathrm{c}}{ }^{2}\right) / 3 \\
& (\Delta / \sigma)_{\max }=0.001 \\
& \Delta \rho_{\max }=0.44 \mathrm{e} \AA^{-3} \\
& \Delta \rho_{\min }=-0.18 \mathrm{e} \AA^{-3}
\end{aligned}
$$

Special details

Experimental. SADABS-2012/1 (Bruker,2012) was used for absorption correction. wR2(int) was 0.1419 before and 0.0438 after correction. The Ratio of minimum to maximum transmission is 0.8088 . The $\lambda / 2$ correction factor is 0.0015 .

Geometry. All e.s.d.'s (except the e.s.d. in the dihedral angle between two 1.s. planes) are estimated using the full

\begin{tabular}{|c|c|c|c|c|}
\hline & $x$ & $y$ & $z$ & $U_{\text {iso }} * / U_{\text {eq }}$ \\
\hline $\mathrm{C} 1$ & $0.36178(4)$ & $0.50978(18)$ & $0.70073(5)$ & $0.01639(17)$ \\
\hline $\mathrm{C} 10$ & $0.44839(4)$ & $0.3697(3)$ & $0.79439(5)$ & $0.0289(2)$ \\
\hline $\mathrm{C} 11$ & $0.14931(4)$ & $0.2622(2)$ & $0.61260(5)$ & 0.02223 (19) \\
\hline $\mathrm{C} 2$ & $0.32768(4)$ & 0.32755 (19) & $0.72601(5)$ & $0.01806(17)$ \\
\hline $\mathrm{C} 3$ & $0.26774(4)$ & $0.30632(18)$ & $0.69042(5)$ & $0.01692(17)$ \\
\hline $\mathrm{C} 4$ & $0.24216(4)$ & $0.47152(18)$ & $0.62979(5)$ & $0.01575(16)$ \\
\hline $\mathrm{C} 5$ & $0.27657(4)$ & $0.65750(18)$ & $0.60495(4)$ & $0.01568(16)$ \\
\hline C6 & $0.33612(4)$ & $0.67778(17)$ & 0.63904 (4) & $0.01468(16)$ \\
\hline $\mathrm{C} 7$ & $0.37340(4)$ & $0.86255(18)$ & $0.60859(5)$ & $0.01664(16)$ \\
\hline $\mathrm{C} 8$ & $0.39755(4)$ & $0.67941(18)$ & $0.56036(5)$ & $0.01553(16)$ \\
\hline $\mathrm{C} 9$ & $0.44172(3)$ & $0.82948(18)$ & $0.53467(4)$ & $0.01464(16)$ \\
\hline $\mathrm{H} 10 \mathrm{~A}$ & 0.4311 & 0.4146 & 0.8315 & $0.043 *$ \\
\hline H10B & 0.4901 & 0.4129 & 0.8124 & $0.043^{*}$ \\
\hline $\mathrm{H} 10 \mathrm{C}$ & 0.4427 & 0.1653 & 0.7820 & $0.043^{*}$ \\
\hline H11A & 0.1506 & 0.3038 & 0.6618 & $0.033^{*}$ \\
\hline H11B & 0.1648 & 0.0690 & 0.6106 & $0.033^{*}$ \\
\hline $\mathrm{H} 11 \mathrm{C}$ & 0.1090 & 0.2724 & 0.5803 & $0.033^{*}$ \\
\hline $\mathrm{H} 2$ & 0.3452 & 0.2162 & 0.7679 & $0.022 *$ \\
\hline H3 & 0.2447 & 0.1796 & 0.7077 & $0.020 *$ \\
\hline $\mathrm{H} 4$ & $0.4791(8)$ & $0.795(4)$ & $0.4682(10)$ & $0.049(5)^{*}$ \\
\hline H5 & 0.2587 & 0.7724 & 0.5638 & $0.019^{*}$ \\
\hline H7A & 0.4059 & 0.9461 & 0.6481 & $0.020 *$ \\
\hline H7B & 0.3498 & 1.0224 & 0.5804 & $0.020 *$ \\
\hline H8A & 0.4156 & 0.5046 & 0.5871 & $0.019 *$ \\
\hline H8B & 0.3647 & 0.6170 & 0.5181 & $0.019 *$ \\
\hline O1 & $0.42100(3)$ & $0.53955(17)$ & $0.73202(4)$ & $0.02384(16)$ \\
\hline $\mathrm{O} 2$ & $0.18373(3)$ & $0.46840(16)$ & 0.59092 (4) & $0.02300(16)$ \\
\hline $\mathrm{O} 3$ & $0.46906(3)$ & $1.04245(15)$ & $0.56342(4)$ & $0.02097(15)$ \\
\hline $\mathrm{O} 4$ & 0.44928 & $0.70388(15)$ & $0.47828(4)$ & $0.02040(15)$ \\
\hline
\end{tabular}
covariance matrix. The cell e.s.d.'s are taken into account individually in the estimation of e.s.d.'s in distances, angles and torsion angles; correlations between e.s.d.'s in cell parameters are only used when they are defined by crystal symmetry. An approximate (isotropic) treatment of cell e.s.d.'s is used for estimating e.s.d.'s involving 1.s. planes.

Fractional atomic coordinates and isotropic or equivalent isotropic displacement parameters $\left(\AA^{2}\right)$ 
Atomic displacement parameters $\left(\AA^{2}\right)$

\begin{tabular}{lllllll}
\hline & $U^{11}$ & $U^{22}$ & $U^{33}$ & $U^{12}$ & $U^{13}$ & $U^{23}$ \\
\hline C1 & $0.0167(4)$ & $0.0191(4)$ & $0.0150(4)$ & $0.0013(3)$ & $0.0074(3)$ & $-0.0003(3)$ \\
C10 & $0.0211(4)$ & $0.0448(6)$ & $0.0197(4)$ & $0.0072(4)$ & $0.0049(3)$ & $0.0065(4)$ \\
C11 & $0.0206(4)$ & $0.0207(4)$ & $0.0263(4)$ & $-0.0063(3)$ & $0.0089(3)$ & $-0.0017(3)$ \\
C2 & $0.0221(4)$ & $0.0190(4)$ & $0.0149(4)$ & $0.0013(3)$ & $0.0086(3)$ & $0.0031(3)$ \\
C3 & $0.0218(4)$ & $0.0159(4)$ & $0.0162(4)$ & $-0.0015(3)$ & $0.0104(3)$ & $0.0008(3)$ \\
C4 & $0.0170(4)$ & $0.0154(3)$ & $0.0162(4)$ & $-0.0009(3)$ & $0.0073(3)$ & $-0.0010(3)$ \\
C5 & $0.0196(4)$ & $0.0144(3)$ & $0.0148(3)$ & $0.0004(3)$ & $0.0081(3)$ & $0.0015(3)$ \\
C6 & $0.0190(4)$ & $0.0130(3)$ & $0.0155(4)$ & $0.0002(3)$ & $0.0103(3)$ & $-0.0010(3)$ \\
C7 & $0.0201(4)$ & $0.0144(3)$ & $0.0200(4)$ & $-0.0012(3)$ & $0.0127(3)$ & $-0.0008(3)$ \\
C8 & $0.0166(4)$ & $0.0159(4)$ & $0.0172(4)$ & $-0.0024(3)$ & $0.0099(3)$ & $-0.0016(3)$ \\
C9 & $0.0137(3)$ & $0.0159(4)$ & $0.0160(3)$ & $0.0007(3)$ & $0.0072(3)$ & $-0.0003(3)$ \\
O1 & $0.0167(3)$ & $0.0334(4)$ & $0.0207(3)$ & $0.0005(3)$ & $0.0053(2)$ & $0.0055(3)$ \\
O2 & $0.0176(3)$ & $0.0258(3)$ & $0.0242(3)$ & $-0.0045(2)$ & $0.0049(3)$ & $0.0060(3)$ \\
O3 & $0.0239(3)$ & $0.0205(3)$ & $0.0238(3)$ & $-0.0077(2)$ & $0.0150(3)$ & $-0.0069(2)$ \\
O4 & $0.0208(3)$ & $0.0229(3)$ & $0.0233(3)$ & $-0.0074(2)$ & $0.0151(3)$ & $-0.0086(2)$ \\
& & & & & & \\
\hline
\end{tabular}

Geometric parameters $\left(\AA,{ }^{\circ}\right)$

\begin{tabular}{llll}
\hline $\mathrm{C} 10-\mathrm{H} 10 \mathrm{C}$ & 0.9800 & $\mathrm{C} 6-\mathrm{C} 5$ & $1.3854(12)$ \\
$\mathrm{C} 10-\mathrm{H} 10 \mathrm{~B}$ & 0.9800 & $\mathrm{C} 7-\mathrm{H} 7 \mathrm{~B}$ & 0.9900 \\
$\mathrm{C} 10-\mathrm{H} 10 \mathrm{~A}$ & 0.9800 & $\mathrm{C} 7-\mathrm{H} 7 \mathrm{~A}$ & 0.9900 \\
$\mathrm{C} 11-\mathrm{H} 11 \mathrm{C}$ & 0.9800 & $\mathrm{C} 8-\mathrm{C} 9$ & $1.5019(11)$ \\
$\mathrm{C} 11-\mathrm{H} 11 \mathrm{~B}$ & 0.9800 & $\mathrm{C} 8-\mathrm{C} 7$ & 0.9900 \\
$\mathrm{C} 11-\mathrm{H} 11 \mathrm{~A}$ & 0.9800 & $\mathrm{C} 8-\mathrm{H} 8 \mathrm{~B}$ & 0.9900 \\
$\mathrm{C} 2-\mathrm{C} 1$ & $1.3882(12)$ & $\mathrm{C} 8-\mathrm{H} 8 \mathrm{~A}$ & $1.4299(12)$ \\
$\mathrm{C} 2-\mathrm{H} 2$ & 0.9500 & $\mathrm{O} 1-\mathrm{C} 10$ & $1.3751(10)$ \\
$\mathrm{C} 3-\mathrm{C} 4$ & $1.3858(12)$ & $\mathrm{O} 1-\mathrm{C} 1$ & $1.4278(11)$ \\
$\mathrm{C} 3-\mathrm{C} 2$ & $1.3985(12)$ & $\mathrm{O} 2-\mathrm{C} 11$ & $1.3756(10)$ \\
$\mathrm{C} 3-\mathrm{H} 3$ & 0.9500 & $\mathrm{O} 2-\mathrm{C} 4$ & $1.2238(10)$ \\
$\mathrm{C} 5-\mathrm{C} 4$ & $1.3995(11)$ & $\mathrm{O} 3-\mathrm{C} 9$ & $0.917(18)$ \\
$\mathrm{C} 5-\mathrm{H} 5$ & 0.9500 & $\mathrm{O} 4-\mathrm{H} 4$ & $1.3224(10)$ \\
$\mathrm{C} 6-\mathrm{C} 1$ & $1.4085(12)$ & $\mathrm{O} 4-\mathrm{C} 9$ & 108.6 \\
$\mathrm{C} 6-\mathrm{C} 7$ & $1.5101(11)$ & & 108.6 \\
& & & $108.5(11)$ \\
$\mathrm{C} 1-\mathrm{C} 2-\mathrm{H} 2$ & 119.7 & $\mathrm{C} 9-\mathrm{C} 8-\mathrm{H} 8 \mathrm{~B}$ & 109.5 \\
$\mathrm{C} 1-\mathrm{C} 2-\mathrm{C} 3$ & $120.65(8)$ & $\mathrm{C} 9-\mathrm{C} 8-\mathrm{H} 8 \mathrm{~A}$ & 109.5 \\
$\mathrm{C} 1-\mathrm{C} 6-\mathrm{C} 7$ & $120.43(8)$ & $\mathrm{C} 9-\mathrm{O} 4-\mathrm{H} 4$ & 109.5 \\
$\mathrm{C} 1-\mathrm{O} 1-\mathrm{C} 10$ & $117.01(7)$ & $\mathrm{H} 10 \mathrm{~A}-\mathrm{C} 10-\mathrm{H} 10 \mathrm{C}$ & 109.5 \\
$\mathrm{C} 2-\mathrm{C} 1-\mathrm{C} 6$ & $120.15(8)$ & $\mathrm{H} 10 \mathrm{~A}-\mathrm{C} 10-\mathrm{H} 10 \mathrm{~B}$ & 109.5 \\
$\mathrm{C} 2-\mathrm{C} 3-\mathrm{H} 3$ & 120.2 & $\mathrm{H} 10 \mathrm{~B}-\mathrm{C} 10-\mathrm{H} 10 \mathrm{C}$ & 109.5 \\
$\mathrm{C} 3-\mathrm{C} 4-\mathrm{C} 5$ & $119.66(8)$ & $\mathrm{H} 11 \mathrm{~A}-\mathrm{C} 11-\mathrm{H} 11 \mathrm{C}$ & 108.2 \\
$\mathrm{C} 3-\mathrm{C} 2-\mathrm{H} 2$ & 119.7 & $\mathrm{H} 11 \mathrm{~A}-\mathrm{C} 11-\mathrm{H} 11 \mathrm{~B}$ & 107.6 \\
$\mathrm{C} 4-\mathrm{C} 5-\mathrm{H} 5$ & 119.3 & $\mathrm{H} 11 \mathrm{~B}-\mathrm{C} 11-\mathrm{H} 11 \mathrm{C}$ & \\
$\mathrm{C} 4-\mathrm{C} 3-\mathrm{C} 2$ & $119.53(8)$ & $\mathrm{H} 7 \mathrm{~A}-\mathrm{C} 7-\mathrm{H} 7 \mathrm{~B}$ & $\mathrm{C} 8-\mathrm{H} 8 \mathrm{~B}$ \\
$\mathrm{C} 4-\mathrm{C} 3-\mathrm{H} 3$ & 120.2 & &
\end{tabular}




$\begin{array}{llll}\mathrm{C} 4-\mathrm{O} 2-\mathrm{C} 11 & 116.09(7) & \mathrm{O} 1-\mathrm{C} 10-\mathrm{H} 10 \mathrm{C} & 109.5 \\ \mathrm{C} 5-\mathrm{C} 6-\mathrm{C} 1 & 118.54(7) & \mathrm{O} 1-\mathrm{C} 10-\mathrm{H} 10 \mathrm{~B} & 109.5 \\ \mathrm{C} 5-\mathrm{C} 6-\mathrm{C} 7 & 120.93(7) & \mathrm{O} 1-\mathrm{C} 10-\mathrm{H} 10 \mathrm{~A} & 109.5 \\ \mathrm{C} 6-\mathrm{C} 7-\mathrm{H} 7 \mathrm{~B} & 109.8 & \mathrm{O} 1-\mathrm{C} 1-\mathrm{C} 2 & 124.15(8) \\ \mathrm{C} 6-\mathrm{C} 7-\mathrm{H} 7 \mathrm{~A} & 109.8 & \mathrm{O} 1-\mathrm{C} 1-\mathrm{C} 6 & 115.71(7) \\ \mathrm{C} 6-\mathrm{C} 7-\mathrm{C} 8 & 109.52(7) & \mathrm{O} 2-\mathrm{C} 11-\mathrm{H} 11 \mathrm{C} & 109.5 \\ \mathrm{C} 6-\mathrm{C} 5-\mathrm{C} 4 & 121.46(8) & \mathrm{O} 2-\mathrm{C} 11-\mathrm{H} 11 \mathrm{~B} & 109.5 \\ \mathrm{C} 6-\mathrm{C} 5-\mathrm{H} 5 & 119.3 & \mathrm{O} 2-\mathrm{C} 11-\mathrm{H} 11 \mathrm{~A} & 115.98(7) \\ \mathrm{C} 7-\mathrm{C} 8-\mathrm{H} 8 \mathrm{~B} & 108.6 & \mathrm{O} 2-\mathrm{C} 4-\mathrm{C} 5 & 124.36(8) \\ \mathrm{C} 7-\mathrm{C} 8-\mathrm{H} 8 \mathrm{~A} & 108.6 & \mathrm{O} 2-\mathrm{C} 4-\mathrm{C} 3 & 124.03(7) \\ \mathrm{C} 8-\mathrm{C} 7-\mathrm{H} 7 \mathrm{~B} & 109.8 & \mathrm{O} 3-\mathrm{C} 9-\mathrm{C} 8 & 122.90(8) \\ \mathrm{C} 8-\mathrm{C} 7-\mathrm{H} 7 \mathrm{~A} & 109.8 & \mathrm{O} 3-\mathrm{C} 9-\mathrm{O} 4 & 113.05(7) \\ \mathrm{C} 9-\mathrm{C} 8-\mathrm{C} 7 & 114.48(7) & \mathrm{O} 4-\mathrm{C} 9-\mathrm{C} 8 & 0.36(12) \\ & & & 179.97(7) \\ \mathrm{C} 1-\mathrm{C} 6-\mathrm{C} 7-\mathrm{C} 8 & 83.52(9) & \mathrm{C} 5-\mathrm{C} 6-\mathrm{C} 1-\mathrm{C} 2 & -92.96(9) \\ \mathrm{C} 1-\mathrm{C} 6-\mathrm{C} 5-\mathrm{C} 4 & -1.12(12) & \mathrm{C} 5-\mathrm{C} 6-\mathrm{C} 1-\mathrm{O} 1 & 0.97(13) \\ \mathrm{C} 10-\mathrm{O} 1-\mathrm{C} 1-\mathrm{C} 2 & 0.15(13) & \mathrm{C} 5-\mathrm{C} 6-\mathrm{C} 7-\mathrm{C} 8 & -179.31(7) \\ \mathrm{C} 10-\mathrm{O} 1-\mathrm{C} 1-\mathrm{C} 6 & -179.45(8) & \mathrm{C} 6-\mathrm{C} 5-\mathrm{C} 4-\mathrm{C} 3 & 19.85(12) \\ \mathrm{C} 11-\mathrm{O} 2-\mathrm{C} 4-\mathrm{C} 5 & 175.08(8) & \mathrm{C} 6-\mathrm{C} 5-\mathrm{C} 4-\mathrm{O} 2 & -161.63(7) \\ \mathrm{C} 11-\mathrm{O} 2-\mathrm{C} 4-\mathrm{C} 3 & -5.21(13) & \mathrm{C} 7-\mathrm{C} 8-\mathrm{C} 9-\mathrm{O} 3 & -176.20(8) \\ \mathrm{C} 2-\mathrm{C} 3-\mathrm{C} 4-\mathrm{C} 5 & -0.03(13) & \mathrm{C} 7-\mathrm{C} 8-\mathrm{C} 9-\mathrm{O} 4 & 3.41(11) \\ \mathrm{C} 2-\mathrm{C} 3-\mathrm{C} 4-\mathrm{O} 2 & -179.73(8) & \mathrm{C} 7-\mathrm{C} 6-\mathrm{C} 1-\mathrm{C} 2 & 175.42(7) \\ \mathrm{C} 3-\mathrm{C} 2-\mathrm{C} 1-\mathrm{C} 6 & \mathrm{C} 7-\mathrm{C} 6-\mathrm{C} 1-\mathrm{O} 1 & -172.25(7) \\ \mathrm{C} 3-\mathrm{C} 2-\mathrm{C} 1-\mathrm{O} 1 & \mathrm{C} 7-\mathrm{C} 6-\mathrm{C} 5-\mathrm{C} 4 & \\ \mathrm{C} 4-\mathrm{C} 3-\mathrm{C} 2-\mathrm{C} 1 & -179.02(8) & \mathrm{C} 9-\mathrm{C} 8-\mathrm{C} 7-\mathrm{C} 6 & \\ & -0.73(13) & & \end{array}$

Hydrogen-bond geometry $\left(\AA,{ }^{\circ}\right)$

\begin{tabular}{lllll}
\hline$D-\mathrm{H} \cdots A$ & $D-\mathrm{H}$ & $\mathrm{H} \cdots A$ & $D \cdots A$ & $D-\mathrm{H} \cdots A$ \\
\hline $\mathrm{O} 4-\mathrm{H} 4 \cdots \mathrm{O}^{\mathrm{i}}$ & $0.92(2)$ & $1.75(2)$ & $2.6624(11)$ & $172.1(18)$ \\
\hline
\end{tabular}

Symmetry code: (i) $-x+1,-y+2,-z+1$. 